\title{
DISTORTION PROPERTIES OF p-FOLD SYMMETRIC ALPHA-STARLIKE FUNCTIONS
}

\author{
H. B. COONCE AND S. S. MILLER ${ }^{1}$
}

Abstract. Starlike functions $f$ which are of Mocanu type $\alpha$ and have power series of the form

$$
f(z)=z+a_{p+1} z^{p+1}+a_{2 p+1} z^{2 p+1}+\cdots,
$$

where $p=1,2,3, \cdots$, are shown to satisfy the relation $f(z)=$ $\left[g\left(z^{p}\right)\right]^{1 / p}$ where $g$ is of Mocanu type $p \alpha$ with power series $g(z)=z+$ $b_{2} z^{2}+b_{3} z^{3}+\cdots$. Distortion results dealing with the 1 -theorem and bounds on $|f(z)|$ are obtained.

1. Introduction. In a recent paper [1] S. S. Miller obtained distortion theorems for the class of alpha-starlike functions. In this paper we look at functions which are alpha-starlike and $p$-fold symmetric. Specifically we look at functions $f$ which are alpha-starlike with power series of the form

$$
f(z)=z+a_{p+1} z^{p+1}+a_{2 p+1} z^{2 p+1}+\cdots,
$$

where $p=1,2,3, \cdots$.

For completeness we recall the pertinent definitions.

Definition 1. Let $\alpha$ be real and suppose $f(z)=z+b_{2} z^{2}+b_{3} z^{3}+\cdots$ is regular in. $D=\{z:|z|<1\}$ with $f(z) f^{\prime}(z) \neq 0$ in $0<|z|<1$. If

$$
\operatorname{Re}\left[(1-\alpha) \frac{z f^{\prime}(z)}{f(z)}+\alpha\left(\frac{z f^{\prime \prime}(z)}{f^{\prime}(z)}+1\right)\right]>0
$$

for $z \in D$ then $f$ is an $\alpha$-starlike function. We write $f \in \mathscr{M}_{\alpha}$.

Definition 2. If $f$ is starlike and $\alpha=\sup \left\{\beta: f \in \mathscr{M}_{\beta}\right\}$ then $f$ is of Mocanu type $\alpha(f \in \mathscr{M}(\alpha))$.

The above definitions may be found in [1], [2] and [3].

We now introduce some notation.

Received by the editors July $10,1972$.

AMS (MOS) subject classifications (1970). Primary 30A32.

Key words and phrases. Starlike, convex, alpha-starlike, Mocanu type, $p$-fold symmetric, distortion theorems.

${ }^{1}$ The second author acknowledges support received under a grant from the Research Foundation of the State of New York.

(c) American Mathematical Society 1974 
Definition 3. If $f \in \mathscr{M}_{\alpha}$ and $f(z)$ has a power series of the form (1.1) we write $f \in \mathscr{M}_{\alpha, p}$. If $f \in \mathscr{M}(\alpha)$ with power series of the form (1.1) we write $f \in \mathscr{M}_{p}(\alpha)$.

The results of this paper will depend upon the theorem (proven in \$2) that $f \in \mathscr{M}_{p}(\alpha)$ iff $g \in \mathscr{M}_{1}(p \alpha)$ where $f(z)=\left[g\left(z^{p}\right)\right]^{1 / p}$. The subsequent distortion theorems (proven in $\S 3$ ) will follow from results in [1].

2. The basic relation. In this section we consider the following result.

THEOREM 1. $f \in \mathscr{M}_{p}(\alpha)$ iff $g \in \mathscr{M}_{1}(p \alpha)$, where $f(z)=\left[g\left(z^{p}\right)\right]^{1 / p}$.

Proof. Let $f \in \mathscr{M}_{\alpha, p}, \alpha$ real, thus

$$
\operatorname{Re}\left\{(1-\alpha) \frac{z f^{\prime}(z)}{f(z)}+\alpha\left(\frac{z f^{\prime \prime}(z)}{f^{\prime}(z)}+1\right)\right\}>0 .
$$

Setting $f(z)=\left[g\left(z^{p}\right)\right]^{1 / p}$ and computing $f^{\prime}(z) / f(z)$ and $f^{\prime \prime}(z) / f^{\prime}(z)$ we notice the left-hand side of $(2.1)$ is equal to

$$
\operatorname{Re}\left\{(1-p \alpha) \frac{z^{p} g^{\prime}\left(z^{p}\right)}{g\left(z^{p}\right)}+p \alpha\left(\frac{z^{p} g^{\prime \prime}\left(z^{p}\right)}{g^{\prime}\left(z^{p}\right)}+1\right)\right\} .
$$

But the condition that this quantity is positive is equivalent to $g \in \mathscr{M}_{p \alpha, 1}$. Since the computations are reversible it follows that $f \in \mathscr{M}_{\alpha, p}$ iff $g \in \mathscr{M}_{p \alpha, 1}$. Furthermore since the correspondence of $\alpha$ and $p \alpha$ is monotone increasing it follows that $f \in \mathscr{M}_{p}(\alpha)$ iff $g \in \mathscr{M}_{1}(p \alpha)$.

Note that an alternate proof to Theorem 1 can be obtained by using the integral representation for functions in $\mathscr{M}_{\alpha}$ (see [1] or [2]) plus the fact that if $g(z)$ is a starlike function then $\left[g\left(z^{n}\right)\right]^{1 / n}$ is also a starlike function.

3. Distortion theorems. In the following theorems we will need the functions

$$
\begin{gathered}
g_{0}(p \alpha, z)=\left[\frac{1}{p \alpha} \int_{0}^{z} \zeta^{1 / p \alpha-1}(1-\zeta)^{-2 / p \alpha} d \zeta\right]^{p \alpha}, \\
f_{0}(\alpha, z)=\left[g_{0}\left(p \alpha, z^{p}\right)\right]^{1 / p}
\end{gathered}
$$

and

$$
K(\alpha, r)=r\left[G\left(\frac{1}{\alpha}, \frac{2}{\alpha}, \frac{1}{\alpha}+1 ; r\right)\right]^{\alpha},
$$

where $G(a, b, c ; z)$ is the hypergeometric function. 
THEOREM 2. If $f(z)$ is a p-fold symmetric alpha-starlike function, $\alpha>0$, then for $|z|=r(0<r<1)$

$$
\left[-K\left(p \alpha,-r^{p}\right)\right]^{1 / p} \leqq|f(z)| \leqq\left[K\left(p \alpha, r^{p}\right)\right]^{1 / p} .
$$

Proof. In [1] it is shown that for $g \in \mathscr{M}_{1}(p \alpha)$,

$$
-K(p \alpha,-r) \leqq|g(z)| \leqq K(p \alpha, r) .
$$

By Theorem $1 f(z)=\left[g\left(z^{p}\right)\right]^{1 / p}$ and (3.4) follows. Since (3.5) is sharp for $g_{0}(p \alpha, z)$, we have equality for $f_{0}(\alpha, z)$.

REMARKS. If $\alpha=1$ and $p=2$ we have for odd convex functions

$$
\tan ^{-1} r \leqq|f(z)| \leqq \frac{1}{2} \log \frac{1+r}{1-r},
$$

whereas if $\alpha$ approaches zero we have the known result for all odd starlike functions

$$
\frac{r}{1+r^{2}} \leqq|f(z)| \leqq \frac{r}{1-r^{2}}
$$

Furthermore, since $g \in \mathscr{M}_{1}(\alpha)$ for $\alpha>2$ implies $g$ is a bounded convex function [1] we have that $f \in \mathscr{M}_{p}(\alpha)$, for $\alpha>2 / p$, is a bounded convex function. In particular all odd alpha-starlike functions are bounded if $\alpha>1$.

THEOREM 3. If $f \in \mathscr{M}_{p}(\alpha), \alpha>0$, with power series (1.1) then $\left|a_{p+1}\right| \leqq 2 / p(1+p \alpha)$ and this bound is sharp.

Proof. In [1] it is shown that if $g \in \mathscr{M}_{1}(p \alpha), p \alpha>0$, the coefficient $b_{2}=g^{\prime \prime}(0) / 2$ satisfies $\left|b_{2}\right| \leqq 2 /(1+p \alpha)$. Since $f(z)=\left[g\left(z^{p}\right)\right]^{1 / p}$, a straightforward calculation shows $\left|a_{p+1}\right| \leqq 2 / p(1+p \alpha)$. This inequality is sharp for $f_{0}$. Notice that for $\alpha=0$ or 1 and $p=2$ this reduces to the familiar bounds 1 and $\frac{1}{3}$ respectively.

THEOREM 4. If $f \in \mathscr{M}_{p}(\alpha), \alpha>0$, then the image of $D$ under the mapping $w=f(z)$ always contains the disc $|w|<\hat{d}(\alpha)$ where

$$
\begin{aligned}
\hat{d}(\alpha) & =\left(\frac{1}{2}\right)^{2 / p} & \text { when } \alpha=0, \\
& =\left[\frac{1}{2 p \alpha} \frac{[\Gamma(1 / p \alpha)]^{2}}{\Gamma(2 / p \alpha)}\right]^{\alpha} & \text { when } \alpha>0 .
\end{aligned}
$$

These results are sharp with equality for $f_{0}$.

Proof. Clearly $\hat{d}(\alpha)=[d(p \alpha)]^{1 / p}$ where $d$ is the radius of the largest disc always contained in the image $w=g(z)$ where $f(z)=\left[g\left(z^{p}\right)\right]^{1 / p}$. But 
by [1],

$$
d(p \alpha)=\left[\frac{1}{2 p \alpha} \frac{\Gamma(1 / p \alpha)^{2}}{\Gamma(2 / p \alpha)}\right]^{p \alpha},
$$

which proves the result for $\alpha>0$. For $\alpha=0$, the Koebe function gives us $\left(\frac{1}{2}\right)^{2 / p}$ and in fact $\lim _{\alpha \rightarrow 0^{+}} \hat{d}(\alpha)=\left(\frac{1}{2}\right)^{2 / p}$, thus establishing the result.

Notice that for $\alpha=0$ or 1 and $p=1$ or 2 , we have $\hat{d}(\alpha)$ given by

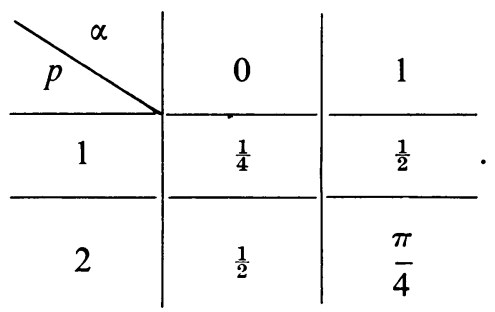

If we let $p \rightarrow \infty$ we notice $\lim _{p \rightarrow \infty} \hat{d}(\alpha)=1, \alpha \geqq 0$ thus providing another proof of the well-known fact that $\lim _{p \rightarrow \infty}\left[g\left(z^{p}\right)\right]^{1 / p}$ is the function $h(z)=z$.

THEOREM 5. If $f \in \mathscr{M}_{p}(\alpha), \alpha>0$, and $M(r)=\max _{\theta}\left|f\left(r e^{i \theta}\right)\right|$, then

$$
\begin{aligned}
M(r) & =O(1 /(1-r))^{(2-p \alpha) / p} & & \text { for } 0 \leqq \alpha<2 / p, \\
& =O \log (1 /(1-r)) & & \text { for } \alpha=2 / p,
\end{aligned}
$$

as $r \rightarrow 1^{-}$. If $\alpha>2 / p$, then

$$
M(r) \leqq\left[\frac{1}{p \alpha} \Gamma(1 / p \alpha) \frac{\Gamma(1-2 / p \alpha)}{\Gamma(1-1 / p \alpha)}\right]^{\alpha} .
$$

These bounds are best possible with equality for $f_{0}$.

Proof. From [1] we see that if $g \in \mathscr{M}_{1}(p \alpha)$, then

$$
\begin{aligned}
\max _{\theta}\left|g\left(r^{p} e^{i \theta}\right)\right| & =O\left(1 /\left(1-r^{p}\right)\right)^{2-p \alpha} & & \text { for } 0 \leqq \alpha<2, \\
& =O\left(\log 1 /\left(1-r^{p}\right)\right) & & \text { for } \alpha=2,
\end{aligned}
$$

as $r \rightarrow 1^{-}$. If $\alpha>2$, then

$$
\max _{\theta}\left|g\left(r^{p} e^{i \theta}\right)\right| \leqq\left[\frac{1}{p \alpha} \frac{\Gamma(1 / p \alpha) \Gamma(1-2 / p \alpha)}{\Gamma(1-1 / p \alpha)}\right]^{p \alpha} .
$$

Letting $f(z)=\left[g\left(z^{p}\right)\right]^{1 / p}$ and taking $p$ th roots of the above we obtain the desired result.

\section{REFERENCES}

1. S. S. Miller, Distortion properties of alpha-starlike functions, Proc. Amer. Math. Soc. 38 (1973), 311-318.

2. S. S. Miller, P. Mocanu and M. O. Reade, All alpha-convex functions are univalent and starlike, Proc. Amer. Math. Soc. 37 (1973), 553-554. 
3. P. T. Mocanu, Une propriété de convexité généralisée dans la théorie de la représentation conforme, Mathematica (Cluj) 11 (34) (1969), 127-133. MR 42 \#7881.

Department of Mathematics, Mankato State College, Mankato, Minnesota 56001 (Current address of H. B. Coonce)

Department of Mathematics, State University of New York, Brockport, NEW YORK 14420

Current address (S. S. Miller): Department of Mathematics, The Babeș-Bolyai University, Cluj, Romania 